\title{
$\checkmark$ Research Square

\section{Can't see the wood for the trees: Using common likelihoods to share information regarding selection of functional form in survival analysis}

Neil Hawkins ( $\nabla$ neil.hawkins@glasgow.ac.uk)

Institute of Health and Wellbeing, University of Glasgow https://orcid.org/0000-0003-3199-221X

Chris Parker

Celgene Ltd

David A. Scott

Diabetes Research Centre, University of Leicester

Technical advance

Keywords: survival analysis, extrapolation, parametric models

Posted Date: August 13th, 2020

DOI: https://doi.org/10.21203/rs.3.rs-55390/v1

License: (c) (i) This work is licensed under a Creative Commons Attribution 4.0 International License.

Read Full License 
1 Can't see the wood for the trees: Using common likelihoods to share information regarding

2 selection of functional form in survival analysis

3

$4 \quad$ Neil Hawkins PhD, ${ }^{1}$ Chris Parker MSc, ${ }^{2}$ David A. Scott $\mathrm{MSc}^{3}$

$5{ }^{1}$ Institute of Health \& Wellbeing, University of Glasgow, 1 Lilybank Gardens, Glasgow, UK

6 neil.hawkins@glasgow.ac.uk [ORCID: 0000-0003-3199-221X]

$7 \quad{ }^{2}$ Celgene Ltd, 1 Longwalk Road, Stockley Park, Uxbridge, UK

8 cparker@celgene.com

$9{ }^{3}$ Diabetes Research Centre, University of Leicester, Leicester General Hospital, Leicester,

10 UK ds647@ leicester.ac.uk [ORCID: 0000-0001-6475-8046] 
Abstract

Background: The estimation of mean survival is a key consideration when estimating the cost-effectiveness of interventions. Estimation typically requires extrapolation using parametric survival analysis and may be sensitive to choice of functional form. We demonstrate a simple method that allows goodness-of-fit information to be assessed jointly across studies, where such information is believed likely to be exchangeable, to help inform the selection of functional form for parametric survival analysis.

Methods: Individual patient data for survival was estimated from digitised Kaplan-Meier curves for four trials in advanced soft tissue sarcoma. A range of parametric survival models were fitted. Two approaches were explored for identifying the preferred parametric model: (i) selecting functional forms independently for each study; and (ii) selecting a common functional form across studies. Akaike's Information Criterion (AIC) or Bayesian Information Criterion (BIC) were used to select optimal functional form. For approach (ii) joint BIC or AIC statistics were calculated for each model by combining the components of the AIC or BIC, respectively, across studies. A bootstrap analysis was conducted to estimate the uncertainty in selection of functional form.

Results: Estimates of mean survival varied markedly according to choice of functional form. Independent selection led to different functional forms being selected for each study with considerable uncertainty regarding the optimum functional form according to AIC or BIC. In this case study, selecting an optimum functional form based on joint AIC or BIC across studies reduced uncertainty in model selection and variance in mean survival compared to selection of functional form independently.

Conclusion: Extrapolation of estimates of survival may be sensitive to choice of functional form. If it is believed, a priori, reasonable to share information regarding the choice of 
44 functional form for survival analysis across studies, estimating joint goodness-of-fit

45 information across studies could reduce uncertainty and lead to more reliable estimates of

46 mean survival and cost-effectiveness.

Keywords: survival analysis, extrapolation, parametric models

\section{Background}

51 Health economic models are an important tool helping decision-makers to identify the most cost-effective health care technologies. These models often use estimates of time-to-event for outcomes such as survival to estimate costs and health outcomes. Typically, a lifetime horizon is appropriate for cost-effective modelling to inform healthcare decision making. In order to estimate lifetime costs and outcomes for all relevant technologies, extrapolation based on parametric survival analysis - beyond the time horizon of observed events in

57 clinical trials is often necessary.

58 It is commonplace to use parametric survival models to estimate mean survival in health

59 technology assessment (HTA). However, estimates of cost-effectiveness may be sensitive to the choice of parametric model. A published review of extrapolation modelling in National

61 Institute for Health and Care Excellence (NICE) technology appraisals identified

62 inconsistencies in the approaches employed for estimating mean survival [1]. This included 63 processes for identification of an appropriate parametric survival function.

64 Recommendations for identification of the most appropriate parametric survival function

65 have been published [1-3]. These recommendations focus on identifying a "preferred" 
parametric survival function from a set of candidates for an individual study and reference the use of external information on expected survival to validate and inform model selection $[4,5]$.

When fitting survival models we often assume that information on the choice of parametric survival function is exchangeable across treatments within a study. In this paper, we extend this notion and assume that parametric survival function is exchangeable across studies within a disease area by sharing information regarding the chosen survival function. Essentially, we identify the best fitting model across the group of studies assuming that the parametric survival function should be the same for all studies. We do this by estimating a common goodness of fit statistics across the studies.

This paper uses a case study in soft tissue sarcoma (STS), for which trials commonly utilise time-to-event endpoints such as overall survival, to illustrate the application of this approach as an extension to currently recommended methods for estimating mean survival. Whilst our example uses clinical trial data, our methods may equally be applied to observational studies or patient registries.

\section{Methods}

\section{Study data}

Soft tissue sarcomas comprise a heterogeneous group of rare tumours accounting for $1 \%$ of all cancers in adults [6]. Approximately 30-40\% of patients diagnosed with STS develop advanced or metastatic disease for which overall survival is poor (median 14-17 months) [7].

Systemic chemotherapy with palliative intent is the only treatment option for the majority of

87 patients with advanced and/or metastatic disease, and as such, overall survival (OS) is a commonly reported endpoint in trials in this population. Treatment with anthracycline-based 
chemotherapy, primarily doxorubicin, either as monotherapy or in combination with ifosfamide, is recommended in first-line. Treatment options after failure of doxorubicin and ifosfamide are limited and there are no standard recognised therapies. Treatments used in

92 clinical practice include; gemcitabine, docetaxel, dacarbazine, and trabectedin $[6,8]$.

93 Our illustration used a set of four clinical trials in advanced soft tissue sarcoma identified

94 from a published systematic review [9]. Six treatments were investigated across four studies

95 [10-13]; two studies were comparative randomised controlled trials (RCTs), and two were 96 non-comparative single arm studies. Each treatment was trialled once. For each study we 97 calculate mean survival for cost-effectiveness analysis by estimating the whole survival curve.

\section{Mean survival estimation}

All studies reported Kaplan-Meier (KM) plots for OS. The published KM curves were digitised using TechDig software [14] to estimate the survival probability at a range of time points. KM curves were cut off at a common timepoint (12 months) to simulate the effects of having limited study follow-up (Figure 1). A published algorithm was then used to estimate the individual patient data (IPD), generating a time-to-event and a censoring variable for each patient [15].

[Figure title] Figure 1: Kaplan-Meier plots for individual studies

[Insert Figure 1 here]

108 [Figure legend] Overall survival in months 
111 Eight parametric survival functions were considered: exponential, gamma, generalised $F$, 112 generalised gamma, Gompertz, lognormal, log-logistic, and Weibull. These represent the 113 commonly used distributions in cost-effectiveness modelling and are the set recommended by

114 NICE [16] with the addition of the generalised $F$ [17], providing additional flexibility with 115 four parameters. For the comparative studies, a joint survival model was fitted to both arms using a treatment dummy covariable.

117 Two approaches were explored for identifying the parametric survival function for a given study; (i) selecting independent parametric survival functions for each study (henceforth referred to as independent selection) and (ii) selecting a common parametric survival function for all studies (henceforth referred to as common selection).

\section{Independent selection}

The parametric survival functions which minimised Akaike's Information Criterion (AIC) or

124 Bayesian Information Criterion (BIC) for each study were chosen as the 'preferred' model.

125 The AIC for a given study $(j)$ and survival model $(m)$ is defined as:

$$
\mathrm{AIC}_{j, m}=-2 \ln \left(L_{j, m}\right)+2 k_{j, m}
$$

126 where $\ln (L)$ is the $\log$-likelihood, $k$ is the number of model parameters, and $n$ is the sample 127 size.

128 The BIC for a given study $(j)$ and survival model $(m)$ was defined as:

$$
\mathrm{BIC}_{j, m}=-2 \ln \left(L_{j, m}\right)+k_{j, m} \ln \left(n_{j}\right)
$$

129 BIC penalises additional parameters more heavily than the AIC for samples relevant to survival analysis $(n \geq 8)$. 
131 For independent selection, a parametric survival function was selected for each study by (i)

132 minimising AIC

$$
m_{j}=\min _{m}\left\{\mathrm{AIC}_{j, m}\right\}
$$

133 and (ii) by minimising BIC,

$$
m_{j}=\min _{m}\left\{\mathrm{BIC}_{j, m}\right\}
$$

134 This approach treats all studies as independent with no information being shared across

135 studies. The survival parameters for each study-specific parametric model $\left(m_{j}\right)$ were then

136 estimated, which provided an estimate of mean survival $(\hat{s})$ for each treatment $(t)$,

$$
\widehat{s_{t}}=S\left(m_{j}\right)=1-F\left(m_{j}\right)
$$

137 where $S\left(m_{j}\right)$ is the survivor function and $F\left(m_{j}\right)$ is the cumulative distribution function.

138 To avoid overestimation of mean survival in the absence of discounting, the survival curve

139 for each treatment was integrated over 15 years based on maximum expected survival in this

140 population.

\section{Common selection}

143 For the common selection approach, a common AIC or BIC is estimated across studies

144 applying the same survival model $m$ to all studies. This was calculated by summing the log

145 likelihood, the number of parameters, and the number of subjects across studies

$$
\begin{gathered}
\mathrm{AIC}_{m}=\sum_{j=1}^{J}-2 \ln \left(L_{j, m}\right)+\sum_{j=1}^{J} 2 k_{j, m} \\
\mathrm{BIC}_{m}=\sum_{j=1}^{J}-2 \ln \left(L_{j, m}\right)+\sum_{j=1}^{J} k_{j, m} \cdot \ln \left(\sum_{j=1}^{J} n_{j}\right)
\end{gathered}
$$


where $J$ is the total number of studies and $m$ is the model being tested. This is analogous to

147 estimating the AIC or BIC for a study by summing the components from the individual study 148 arms.

149 The preferred functional form for the survival model is identified by minimising the 150 ‘common’ AIC or BIC.

$$
\begin{aligned}
& M_{A I C}=\min _{m}\left\{\mathrm{AIC}_{m}\right\} \\
& M_{B I C}=\min _{m}\left\{\mathrm{BIC}_{m}\right\}
\end{aligned}
$$

151 The survival parameters for the preferred parametric model were estimated individually for each study which provided an estimate of mean survival for each treatment,

$$
\widehat{S_{t}}=S(M)=1-F(M)
$$

\section{Bootstrap}

154 The variance in the estimated mean and uncertainty in the choice of parametric survival function were estimated for each study under both methods of selection using a bootstrap analysis [18]. The bootstrap analysis was conducted by taking one thousand samples with

157 replacement from the IPD for each study. Within each bootstrap sample $(i)$, the preferred 158 parametric survival function was chosen using both methods of selection:

- Independent selection,

$$
\begin{gathered}
m_{j, i}=\min _{m}\left\{\mathrm{AIC}_{j, m, i}\right\} \text { or } \\
m_{j, i}=\min _{m}\left\{\mathrm{BIC}_{j, m, i}\right\}
\end{gathered}
$$

$$
\widehat{s_{t, l}}=S\left(m_{j, i}\right)
$$


162

163

164

165

166

167

- Common selection,

$$
\begin{gathered}
M_{i}=\min _{m}\left\{\mathrm{AIC}_{m, i}\right\} \text { or } \\
M_{i}=\min _{m}\left\{\mathrm{BIC}_{m, i}\right\}
\end{gathered}
$$

64

$$
\widehat{S_{t, l}}=S\left(M_{i}\right)
$$

The uncertainty in mean survival was obtained from the variance of estimated means across samples. Uncertainty in parametric survival function selection was estimated based on the proportion of bootstrapped samples for which each parametric survival function was preferred under each method of selection.

\section{Kaplan-Meier approach}

We also compared the individual and common selection methods to a "Kaplan-Meier" approach. The Kaplan Meier sample average estimate was used to estimate survival until the timepoint at which $80 \%$ of observed events had occurred. Subsequently, a fully parametric model with an exponential survival function was used. The exponential models were fitted using data from the cut-point onwards. They included a covariable representing treatment for the parallel trials. A similar approach, referred to as the Kaplan-Meier-exponential method, has been described in the related literature [16].

\section{Results}


The parametric survival function associated with the lowest AIC (Table 1) or BIC (Table 2) varied across studies. For AIC using independent selection, the lognormal, log-logistic, loglogistic, and gamma survival functions were selected for studies 1 to 4 , respectively. Using common selection across studies, the log-logistic survival function had the lowest AIC. For BIC using independent selection, the exponential, exponential, log-logistic, and gamma survival functions were selected for studies 1 to 4 , respectively. Using a common selection across studies, the exponential survival function had the lowest BIC.

Table 1: AIC statistics

\begin{tabular}{lrrrrr}
\hline $\begin{array}{l}\text { Parametric survival } \\
\text { function }\end{array}$ & Study 1 & Study 2 & Study 3 & Study 4 & Common \\
\hline \hline Exponential & 456.6 & 431.5 & 368.7 & 1422.9 & 2679.6 \\
Gamma & 458.6 & 432.3 & 358.8 & $\underline{\mathbf{1 4 0 4 . 8}}$ & 2654.5 \\
Generalised F & 460.3 & 434.7 & 361.5 & 1408.4 & 2664.9 \\
Generalised gamma & 458.1 & 433.2 & 360.7 & 1405.8 & 2657.7 \\
Gompertz & 458.3 & 433.4 & 361.2 & 1412.6 & 2665.6 \\
Log-logistic & 457.9 & $\underline{\mathbf{4 3 1}}$ & $\underline{\mathbf{3 5 8 . 6}}$ & 1404.9 & $\mathbf{2 6 5 2 . 4}$ \\
Log-normal & $\mathbf{4 5 6 . 2}$ & 431.8 & 362.2 & 1405.6 & 2655.8 \\
Weibull & $\mathbf{4 5 8 . 5}$ & 432.7 & 358.7 & 1405.9 & 2655.8 \\
\hline
\end{tabular}

Bold underlined values are for preferred parametric survival function

Table 2: BIC statistics

\begin{tabular}{lrrrrr}
\hline $\begin{array}{l}\text { Parametric survival } \\
\text { function }\end{array}$ & Study 1 & Study 2 & Study 3 & Study 4 & Common \\
\hline \hline Exponential & $\underline{\mathbf{4 5 9 . 5}}$ & $\underline{\mathbf{4 3 6 . 8}}$ & 371.4 & 1430.7 & $\underline{\mathbf{2 7 8 9 . 1}}$ \\
Gamma & 464.4 & 440.4 & 364.3 & $\underline{\mathbf{1 4 1 6 . 5}}$ & 2837 \\
Generalised F & 471.9 & 448.1 & 372.5 & 1428 & 2993.4 \\
Generalised gamma & 466.8 & 443.9 & 368.9 & 1421.4 & 2913.2 \\
Gompertz & 464.1 & 441.5 & 366.7 & 1424.3 & 2848.1 \\
Log-logistic & 463.7 & 439.1 & $\underline{\mathbf{3 6 4 . 1}}$ & 1416.6 & 2834.9 \\
Log-normal & 462 & 439.9 & 367.7 & 1417.3 & 2838.3 \\
Weibull & 464.4 & 440.7 & 364.2 & 1417.7 & 2838.3 \\
\hline
\end{tabular}

190 Bold underlined values are for preferred parametric survival function

191 Table 3 presents the mean survival predictions for each treatment based on the candidate

192 parametric survival functions as well as the Kaplan-Meier approach for a single sample. The 
choice of parametric survival function influenced the mean survival predictions; the withinstudy variation in mean survival between parametric survival functions ranged from 7.7 months (study 4, treatment 2) to 13.9 months (study 3 , treatment 1 ).

Table 3: Mean survival predictions for each parametric survival function from a single sample (months)

\begin{tabular}{|c|c|c|c|c|c|c|}
\hline \multirow[b]{2}{*}{$\begin{array}{l}\text { Parametric survival } \\
\text { function }\end{array}$} & Study 1 & \multicolumn{2}{|l|}{ Study 2} & Study 3 & \multicolumn{2}{|l|}{ Study 4} \\
\hline & Treat 1 & Treat 1 & Treat 2 & Treat 1 & Treat 1 & Treat 2 \\
\hline Exponential & 22.81 & 12.48 & 20.8 & 19.75 & 18.49 & 16.4 \\
\hline Gamma & 23.02 & 11.75 & 18.84 & 14.35 & $\underline{15.27}$ & $\underline{13.77}$ \\
\hline Generalised F & 40.52 & 23.31 & 32.13 & 25.57 & 19.33 & 17.19 \\
\hline $\begin{array}{l}\text { Generalised } \\
\text { gamma }\end{array}$ & 39.16 & 14.52 & 24.43 & 13.55 & 17.69 & 15.57 \\
\hline Gompertz & 33.57 & 12 & 19.29 & 11.7 & 12.76 & 11.85 \\
\hline Log-logistic & 33.79 & $\underline{17.39}$ & 26.43 & 18.33 & 21.01 & 18.89 \\
\hline Log-normal & $\underline{36.32}$ & 17.63 & 29.27 & 20.48 & 22.16 & 19.05 \\
\hline Weibull & 23.45 & 11.63 & 18.58 & 13.26 & 14.46 & 13.17 \\
\hline Kaplan-Meier/Exp & 26.68 & 23.05 & 28.91 & 21.72 & 21 & 19.16 \\
\hline
\end{tabular}

Underlined data show preferred parametric survival function under independent selection using AIC, bold data show preferred parametric survival function under independent selection using BIC, and highlighted data show preferred parametric survival function under joint selection using AIC (log-logistic) and BIC (exponential).

There was considerable uncertainty regarding the choice of parametric survival function. The bootstrap estimation for the probability that the preferred parametric survival function was selected varied from $20 \%$ to $45 \%$ across studies for the AIC (Table 4 ) and between $12 \%$ and $69 \%$ for the BIC (Table 5). For example, whilst fitting each of the models to the trial data showed the log-logistic was the best model choice for study 3 using AIC, the bootstrapped estimates revealed the Gompertz, log-normal and Weibull also had relatively high probabilities of being the best model choice (Table 4). To illustrate the uncertainty, Figure 2 shows 16 bootstrapped samples of Study 3 and the best fitting model under the AIC in each case. 
211 Using common selection across studies, the estimated probability that the log-logistic model

212 was chosen with the AIC was estimated as $29 \%$ and the estimated probability that the

213 exponential model was chosen was estimated as $100 \%$ with the BIC. More specifically,

214 sharing goodness-of-fit information across studies reduced the estimated uncertainty in model

215 selection.

216 Table 4: Bootstrap estimated probabilities of uncertainty in model choice (AIC)

\begin{tabular}{lrrrrr}
\hline $\begin{array}{l}\text { Parametric survival } \\
\text { function }\end{array}$ & Study 1 & Study 2 & Study 3 & Study 4 & Common \\
\hline \hline Exponential & $26 \%$ & $22 \%$ & $0 \%$ & $0 \%$ & $0 \%$ \\
Gamma & $0 \%$ & $1 \%$ & $7 \%$ & $\underline{\mathbf{3 4 \%}}$ & $8 \%$ \\
Generalised $F$ & $1 \%$ & $12 \%$ & $6 \%$ & $0 \%$ & $4 \%$ \\
Generalised gamma & $24 \%$ & $6 \%$ & $0 \%$ & $0 \%$ & $22 \%$ \\
Gompertz & $2 \%$ & $1 \%$ & $13 \%$ & $0 \%$ & $0 \%$ \\
Log-logistic & $0 \%$ & $\underline{\mathbf{2 0 \%}}$ & $\underline{\mathbf{3 0 \%}}$ & $17 \%$ & $\underline{\mathbf{2 9 \%}}$ \\
Log-normal & $\mathbf{4 5 \%}$ & $37 \%$ & $22 \%$ & $39 \%$ & $31 \%$ \\
Weibull & $1 \%$ & $1 \%$ & $23 \%$ & $9 \%$ & $6 \%$ \\
\hline
\end{tabular}

217 Bold underlined data are for preferred parametric survival function

218 Table 5: Bootstrap estimated probabilities of uncertainty in model choice (BIC)

\begin{tabular}{lrrrrr}
\hline $\begin{array}{l}\text { Parametric survival } \\
\text { function }\end{array}$ & Study 1 & Study 2 & Study 3 & Study 4 & Common \\
\hline \hline Exponential & $\underline{\mathbf{6 9 \%}}$ & $\underline{\mathbf{5 8 \%}}$ & $5 \%$ & $0 \%$ & $\underline{\mathbf{1 0 0 \%}}$ \\
Gamma & $0 \%$ & $0 \%$ & $23 \%$ & $\underline{\mathbf{3 6 \%}}$ & $0 \%$ \\
Generalised $F$ & $24 \%$ & $30 \%$ & $22 \%$ & $0 \%$ & $0 \%$ \\
Generalised gamma & $0 \%$ & $6 \%$ & $31 \%$ & $0 \%$ & $0 \%$ \\
Gompertz & $0 \%$ & $0 \%$ & $6 \%$ & $0 \%$ & $0 \%$ \\
Log-logistic & $0 \%$ & $0 \%$ & $\underline{\mathbf{1 2 \%}}$ & $17 \%$ & $0 \%$ \\
Log-normal & $5 \%$ & $3 \%$ & $0 \%$ & $39 \%$ & $0 \%$ \\
Weibull & $1 \%$ & $2 \%$ & $0 \%$ & $8 \%$ & $0 \%$ \\
\hline
\end{tabular}

219 Bold underlined data are for preferred parametric survival function

220 [Figure 2 title] Figure 2: Sixteen bootstrapped samples from Study 3 and best fitting model

221 under each sample

222 [Insert Figure 2 here] 
223 The standard error associated with the mean survival estimates was reduced when using

224 bootstrapped common selection compared to both independent selection and the Kaplan-

225 Meier method (Table 6). Sharing goodness-of-fit information across studies to identify a

226 preferred common model can therefore reduce uncertainty in survival estimates. Table 6

227 shows the averages across the 1000 bootstrapped samples thus will not correspond to Table 3

228 which comprises on a single sample.

229 Table 6: Survival predictions averaged over bootstrapped samples (months)

\begin{tabular}{|c|c|c|c|c|c|c|}
\hline \multirow{2}{*}{\multicolumn{2}{|c|}{ 胥 }} & $\begin{array}{c}\text { Kaplan-Meier/ } \\
\text { exponential }\end{array}$ & $\begin{array}{c}\text { AIC } \\
\text { Independent } \\
\text { selection }\end{array}$ & $\begin{array}{c}\text { AIC } \\
\text { Common } \\
\text { selection }\end{array}$ & $\begin{array}{c}\text { BIC } \\
\text { Independent } \\
\text { selection }\end{array}$ & $\begin{array}{c}\text { BIC } \\
\text { Common } \\
\text { selection }\end{array}$ \\
\hline & & $\begin{array}{c}\text { Mean } \\
\text { (SE) }\end{array}$ & $\begin{array}{c}\text { Mean } \\
\text { (SE) }\end{array}$ & $\begin{array}{c}\text { Mean } \\
\text { (SE) }\end{array}$ & $\begin{array}{c}\text { Mean } \\
\text { (SE) }\end{array}$ & $\begin{array}{c}\text { Mean } \\
\text { (SE) }\end{array}$ \\
\hline
\end{tabular}

\begin{tabular}{|c|c|c|c|c|c|c|}
\hline 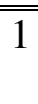 & 1 & $\begin{array}{l}32.07 \\
(7.59)\end{array}$ & $\begin{array}{c}35.76 \\
(12.13)\end{array}$ & $\begin{array}{c}34.97 \\
(10.04)\end{array}$ & $\begin{array}{l}28.19 \\
(9.56)\end{array}$ & $\begin{array}{c}23.1 \\
(2.95)\end{array}$ \\
\hline 2 & 1 & $\begin{array}{l}22.91 \\
(4.88)\end{array}$ & $\begin{array}{l}18.18 \\
(6.63)\end{array}$ & $\begin{array}{l}17.49 \\
(5.48)\end{array}$ & $\begin{array}{l}14.95 \\
(5.03)\end{array}$ & $\begin{array}{l}12.93 \\
(2.29)\end{array}$ \\
\hline 2 & 2 & $\begin{array}{c}29.3 \\
(5.97)\end{array}$ & $\begin{array}{l}27.34 \\
(7.57)\end{array}$ & $\begin{array}{l}26.39 \\
(6.71)\end{array}$ & $\begin{array}{l}23.69 \\
(6.18)\end{array}$ & $\begin{array}{l}21.31 \\
(4.31)\end{array}$ \\
\hline 3 & 1 & $\begin{array}{l}25.24 \\
(8.52)\end{array}$ & $\begin{array}{l}16.64 \\
(4.56)\end{array}$ & $\begin{array}{l}16.38 \\
(3.04)\end{array}$ & $\begin{array}{l}16.14 \\
(3.75)\end{array}$ & $\begin{array}{l}20.02 \\
(2.59)\end{array}$ \\
\hline 4 & 1 & $\begin{array}{l}20.23 \\
(2.61)\end{array}$ & $\begin{array}{l}16.73 \\
(3.55) \\
\end{array}$ & $\begin{array}{l}16.56 \\
(2.66) \\
\end{array}$ & $\begin{array}{l}16.72 \\
(3.44) \\
\end{array}$ & $\begin{array}{l}16.64 \\
(2.05)\end{array}$ \\
\hline 4 & 2 & $\begin{array}{c}21.7 \\
(2.49)\end{array}$ & $\begin{array}{l}18.75 \\
(4.08)\end{array}$ & $\begin{array}{l}18.51 \\
(3.08)\end{array}$ & $\begin{array}{l}18.75 \\
(4.01)\end{array}$ & $\begin{array}{l}18.49 \\
(1.68)\end{array}$ \\
\hline
\end{tabular}

230

231 Discussion

232 These results demonstrate that, in our case study, mean survival estimates are sensitive to

233 which parametric survival function is selected. The choice of parametric survival function

234 based on AIC or BIC was found to be uncertain leading to uncertainty in estimates of mean

235 survival and hence any cost-effectiveness estimates based on these estimates. Our findings 
are in agreement with the current debate around the process in choosing an appropriate

237 parametric survival function and thereby informing economic evaluation [1-3].

238 In our case study, this simple method of sharing goodness-of-fit information across studies reduced the uncertainty in both parametric survival function selection and the corresponding estimates of mean survival compared to independent selection. The utility of this approach for other analysis will depend on whether it is believed, a priori, reasonable to share information regarding the choice of functional form for survival analysis across studies. If it is, estimating joint goodness-of-fit information across studies could reduce uncertainty and lead to more reliable estimates of mean survival and cost-effectiveness. More complex models for sharing information regarding functional form exist that allow for uncertainty in the choice of functional form [19] and should also be considered.

247 We utilised the Guyot algorithm [15] to estimate IPD from KM curves, a new technique when Latimer [1,3] and Bagust and Beale [2] entered the debate over methods for extrapolation of trial data. With the widespread use of this algorithm enabling estimation of a IPD from published studies, this debate is more important than ever.

Typically, a lifetime horizon is appropriate for economic modelling to inform healthcare decision making. The observed study data therefore need to be "extrapolated". Whilst external information such as administration data, registries, or expert opinion has been used for validation of extrapolation, recent approaches have proposed its use to inform extrapolation [4,5]. Guyot et al [4] proposed using external information, such as registries, to inform model extrapolation through the use of polynomial functions and population matching

257 techniques. This raises questions about the availability and choice of such data, obtaining access, heterogeneity compared to the study population, missing data, and analysis complexity. 
On the principle of parsimony, we aimed to identify simpler parametric models in preference

261

262

263

264

265

266

267

268

269

270

271

272

273

274

to more complex models which utilise additional parameters to fit to the observed data. We nevertheless used both AIC and BIC methods for identifying the preferred parametric survival function. The BIC discounts the goodness-of-fit of more complex models more heavily compared to AIC.

Uncertainty in the data used in this analysis supports the use of simpler more generalisable models, which are more likely to be identified by BIC. However, there may be other measures and wider criteria which could be considered. This contrasts with Jackson et al [20] who cite the predictive ability of the AIC makes it more appropriate in a health economic modelling context.

The parametric functions included in the set of candidates specified cover proportional hazards and accelerated failure time metrics. Recommendations for identifying a preferred parametric model state the need to assess the plausibility of the proportional hazards assumption, which when violated should lead to proportional hazards models being removed from the set of candidates [1]. Such an approach could be implemented alongside the approaches presented here.

\section{Limitations}

Bagust and Beale [2] describe aspects of RCTs which prevent a good model fit being obtained. These include the mode of action of treatments, confounding by post-protocol therapies and heterogeneity. These could all differ across studies, and our approach does directly not account for this. However, we feel sharing of goodness-of-fit information across studies is a useful adjunct to the existing recommendations for model selection [1,2]. 
Use of AIC or BIC for identifying the preferred parametric survival function only ranks the set of candidate models based on within-trial fit, hence does not consider long term extrapolation. Ensuring long term extrapolations are clinically plausible is a key consideration when modelling survival data, and as such, we would recommend applying the same assessment to the survival predictions obtained with independent and common selection by removing models providing implausible extrapolations from the set of candidates.

A potentially limiting underlying assumption is that the preferred model is among the set of candidate models [2,21]. Inevitably, none of the models may be suitable for the dataset under consideration, however the set considered in this analysis includes all of the parametric survival models available in commonly used statistical packages and that are recommended by the NICE Decision Support Unit [1]. In addition, we have included the four-parameter generalised $F$, an umbrella distribution containing the exponential, Weibull, lognormal, loglogistic, and gamma distributions [22,23], hence the set of candidate models is comprehensive in its ability to model a variety of hazard functions.

\section{Conclusions}

The process for conducting parametric survival analysis for cost-effectiveness analysis has been widely discussed, and recommendations for identifying the most appropriate parametric models have been presented in the literature. These recommendations focus on identifying a "preferred" parametric survival function from the set of candidates, and treat individual studies included in the analysis independently.

However, we have demonstrated that sharing of goodness-of-fit information across studies, if this is believed to reasonable a priori, can potentially enhance this model selection process by reducing uncertainty in structural assumptions. 
306 Given the considerable uncertainty in selecting a parametric survival function and the

307 influence of this process on mean survival estimates, these methods could be used to reduce

308 uncertainty in the selection of parametric survival function and lead to more reliable

309 estimates of mean survival and cost-effectiveness.

310 The data that support the findings of this study are available from the corresponding author

311 upon reasonable request.

313 Abbreviations: Bayesian Information Criterion (BIC); Akaike's Information Criterion

314 (AIC); health technology assessment (HTA); Kaplan-Meier (KM); National Institute for

315 Health and Care Excellence (NICE); overall survival (OS); randomised controlled trials

316 (RCTs); soft tissue sarcoma (STS)

\section{Declarations:}

319 Ethics approval and consent to participate: Not applicable

320 Consent for publication: All authors consent to publication

321 Availability of data and materials: The datasets analysed during the current study are

322 available from the corresponding author on reasonable request.

323 Competing interests: None

324 Funding: DAS acknowledges the support of NIHR Collaboration for Leadership in Applied

325 Health Research and Care East Midlands (CLAHRC EM), UK. The funding agreement

326 ensured the author's independence in designing the study, interpreting the data, writing, and

327 publishing the report. $\mathrm{CP}$ and $\mathrm{NH}$ did not receive any funding. 
328 Authors' contributions: NH, CP and DAS all contributed to analysis and drafting of this manuscript

Acknowledgements: NH would like to thank his friends, family, acquaintances

\section{References}

[1] Latimer NR. Survival analysis for economic evaluations alongside clinical trials-extrapolation with patient-level data: inconsistencies, limitations, and a practical guide. Medical Decision Making 2013; 33(6):743-54

[2] Bagust A, Beale S. Survival analysis and extrapolation modeling of time-to-event clinical trial data for economic evaluation: an alternative approach. Medical Decision Making $2014 ; 34(3): 343-51$

[3] Latimer NR. Response to "Survival analysis and extrapolation modeling of time-to-event clinical trial data for economic evaluation: an alternative approach" by Bagust and Beale. Medical Decision Making 2014; 34(3):279-82

[4] Guyot P, Ades AE, Beasley M, Lueza B, Pignon JP, Welton NJ. Extrapolation of Survival Curves from Cancer Trials Using External Information. Medical Decision Making 2017;37(4): 353-366

[5] Jackson C, Stevens J, Ren S, Latimer N, Bojke L, Manca A, Sharples L. Extrapolating survival from randomized trials using external data: a review of methods. Medical Decision Making 2017; 37:377-390

[6] von Mehren M, Randall RL, Benjamin RS, et al. Soft Tissue Sarcoma, Version 2.2016, NCCN Clinical Practice Guidelines in Oncology. Journal of the National Comprehensive Cancer Network 2016; 14:758-86 
[7] The ESMO / European Sarcoma Network Working Group. Soft tissue and visceral sarcomas: ESMO Clinical Practice Guidelines for diagnosis, treatment and follow-up. Annals of Oncology 2012; 23 (Supplement 7): vii92-99

[8] Frezza AM, Stacchiotti S, Gronchi A. Systemic treatment in advanced soft tissue sarcoma: what is standard, what is new. BMC Medicine 2017; 15:109

[9] Sharma S, Takyar S, Manson SC, Powell S, Penel N. Efficacy and safety of pharmacological interventions in second- or later-line treatment of patients with advanced soft tissue sarcoma: a systematic review. BMC Cancer 2013; 13:385

[10] Demetri GD, Chawla SP, von MM, et al. Efficacy and safety of trabectedin in patients with advanced or metastatic liposarcoma or leiomyosarcoma after failure of prior anthracyclines and ifosfamide: results of a randomized phase II study of two different schedules. J Clin Oncol 2009; 27(25):4188-4196

[11] Garcia-Del-Muro X, Lopez-Pousa A, Maurel J, et al. Randomized phase II study comparing gemcitabine plus dacarbazine versus dacarbazine alone in patients with previously treated soft tissue sarcoma: a Spanish Group for Research on Sarcomas study. J Clin Oncol 2011; 29(18):2528-2533.

[12] Nielsen OS, Judson I, van HQ, et al. Effect of high-dose ifosfamide in advanced soft tissue sarcomas. A multicentre phase II study of the EORTC Soft Tissue and Bone Sarcoma Group. Eur J Cancer 2000; 36(1):61-67

[13] van der Graaf WT, Blay JY, Chawla SP, et al. Pazopanib for metastatic soft-tissue sarcoma (PALETTE): a randomised, double-blind, placebo-controlled phase 3 trial. Lancet 2012; 379(9829):1879-1886

[14] Techdig. Available at http://www.thetechdig.net/ 
[15] Guyot P, Ades AE, Ouwens MJNM, Welton NJ. Enhanced secondary analysis of survival data: reconstructing the data from published Kaplan-Meier survival curves. BMC Medical Research Methodology 2012; 12:9

[16] Latimer N. NICE DSU Technical Support Document 14: Undertaking survival analysis for economic evaluations alongside clinical trials — extrapolation with patientlevel data. 2011. Available from http://www.nicedsu.org.uk

[17] Cox C. The generalized $F$ distribution: An umbrella for parametric survival analysis. Statistics in Medicine 2008; 27:4301-12

[18] Bland MJ, Altman DG. Statistics Notes: Bootstrap resampling methods. BMJ 2015; $350: \mathrm{h} 2622$

[19] Murray, Thomas A., Brian P. Hobbs, and Bradley P. Carlin. 'Combining Nonexchangeable Functional or Survival Data Sources in Oncology Using Generalized Mixture Commensurate Priors'. The Annals of Applied Statistics 9, no. 3 (September 2015): 1549-70. https://doi.org/10.1214/15-AOAS840

[20] Jackson CH, Thompson SG, Sharples LD. Accounting for uncertainty in health economic decision models using model averaging. Journal of the Royal Statistical Society A $2009 ; 172: 383-404$

[21] Wagenmakers E-J, Farrell S. AIC model selection using Akaike weights. Psychonomic Bulletin \& Review 2004, 11:192-196

[22] Jackson CH, Sharples LD, Thompson SG. Survival Models in Health Economic Evaluations: Balancing Fit and Parsimony to Improve Prediction. International Journal of Biostatistics 2010; 6(1):34

[23] Collett D. Modelling Survival Data in Medical Research. Second Edition. CRC Press: London, 2003 
Figures
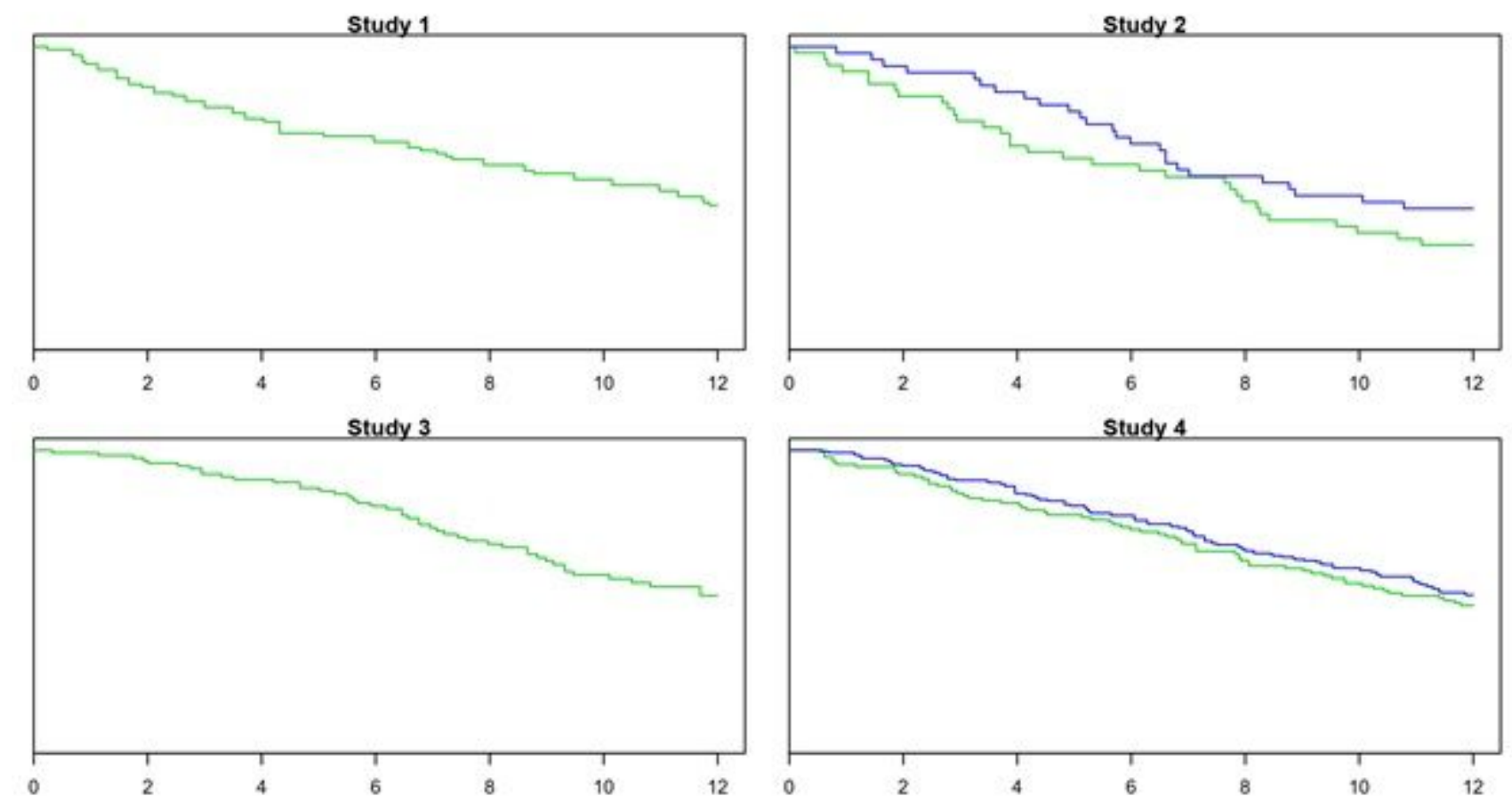

Figure 1

Kaplan-Meier plots for individual studies Overall survival in months 

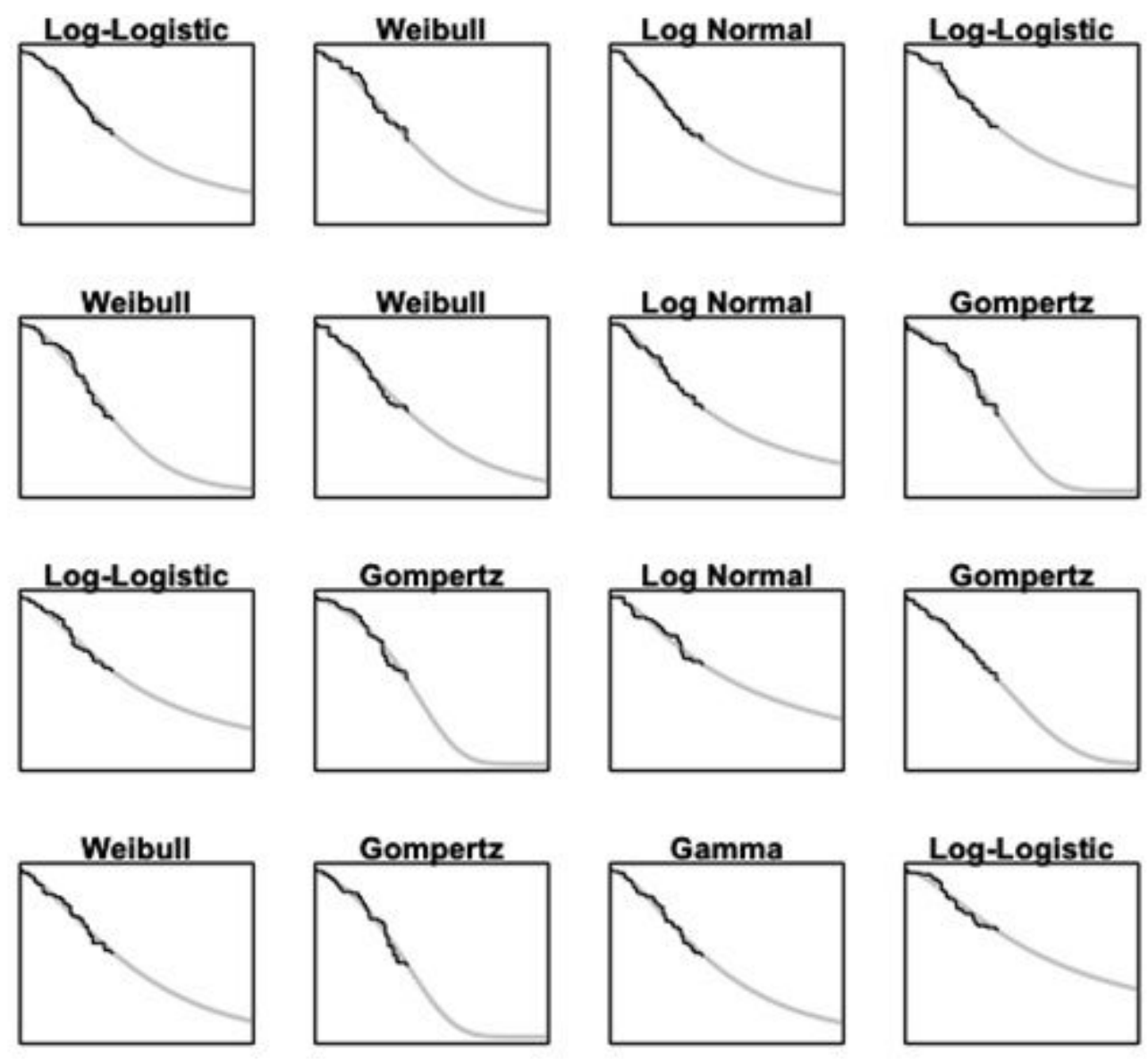

Figure 2

Sixteen bootstrapped samples from Study 3 and best fitting model under each sample 